\title{
La filosofia nacque in Egitto? Le fonti storiche nell'interpreta- zione dell'Afrocentrismo e dei suoi critici
}

$D i$

\section{Luca Bussotti}

CEC, Maputo (Mozambique)

e ISGE-GM, Marracuene (Mozambique)

$\mathrm{L}$ 'Afrocentrismo è una corrente filosofica contemporanea afro-americana, il cui fondatore è Molefi Kete Asante, filosofo afro-americano di origini ghanesi e iniziatore del primo dottorato di ricerca in Studi Afro-Americani presso la Temple University a Philadelphia, nel 1987. Asante rappresenta il pensatore che ha compiuto il tentativo più organico di superare il paradigma eurocentrico per fondarne uno di tipo afrocentrico, sulla base di studi a lui precedenti, che affondano le loro radici su due filoni fondamentali: da un lato, la tradizione egizia, di cui si era occupato, sin dagli anni Cinquanta, il francosenegalese Cheick Anta Diop e, poco dopo, Théophile Obenga, studioso franco-congolese; parallelamente, George James presentava al pubblico un libro assai polemico, preso a riferimento da gran parte dell'attuale corrente afrocentrica, Stolen Legacy (1954), in cui si teorizzava il «furto» che i 
filosofi greci avevano perpetrato nei confronti dei veri iniziatori del pensiero filosofico, i sacerdoti egizi. Dall'altro, la tradizione relativa al pensiero africano contemporaneo, con valutazioni di diversa natura fatte da Asante su intellettuali quali Du Bois, Washington, Garvey, Luter King e molti altri, che qui non verrà trattata.

Attraverso la revisione e la rielaborazione di queste due tradizioni, Asante dà origine a quello che lui stesso ritiene essere un nuovo paradigma, l'Afrocentrismo (Afrocentricity), con testi che, intorno agli anni Ottanta, ripropongono in maniera assai esplicita e in certo senso azzardata le tematiche care a Diop, Obenga e James, scuotendo il dibattito accademico e, in generale, pubblico negli Stati Uniti, e registrando crescenti successi presso buona parte della comunità afro-americana e di qualche gruppo di ricerca propriamente africano.

I testi che costituiscono il manifesto dell'Afrocenstrismo possono essere considerati Afrocentricity (1980) e The Afrocentric idea (1987) e, immediatamente a seguire, Kemet, Afrocentricity and Knowledge (1990), in cui Asante, per la prima volta in forma così dettagliata, sposa le tesi proposte da Stolen Legacy, approfondendo soprattutto i contenuti e gli insegnamenti presumibilmente trasmessi dai sacerdoti dei grandi misteri egizi ai filosofi greci, a partire da Talete. In seguito, Asante pubblicherà altri testi di filosofia egizia, ${ }^{1}$ sulla base degli studi di Anta Diop e Obenga, ${ }^{2}$ ma con la partecipazione di altri, significativi autori, quali Maulana Karenga. ${ }^{3}$

L'Afrocentrismo è, secondo la definizione classica data dallo stesso Asante, " a mode of thought and action in which the centrality of African interests, values, and perspectives predominate $» .{ }^{4} \mathrm{Si}$ tratta di un punto di vista eminentemente essenzialista, in cui la «blackness » ricopre un ruolo centrale, e in cui saranno cinque le tappe che dovranno essere percorse, individualmente e collettivamente, dal «black people », al fine di ottenere la definitiva liberazione, fisica e mentale, rispetto alle catene coloniali e alla schiavitù contemporanea che ancora caratterizza la condizione degli africani, sia in continente che nella diaspora.

${ }^{1}$ Cf. M. K. Asante, Kemet, 1990, e M. K. Asante, The Egyptian Philosophers, 2000. Vedere i riferimenti completi delle opera citate in bibliografia.

${ }^{2}$ Cf., fra le altre opere, C. A. DIop, The African Origin of Civilization, 1974, e T. OBENGA,, La philosophie africaine de la période pharaonique. 2780-330 avant notre ère, 1990.

${ }^{3}$ M. KARENGA, MAAT. The Moral Idea of Ancient Egypt, 2004.

${ }^{4}$ M.K. ASANTE, Afrocentricity, 2003, p. 2. 
Per rendere crerdibile il suo disegno filosofico e ideologico, Asante deve dimostrare almeno due elementi: in primo luogo, considerare la civiltà africana come un tutto organico e omogeneo; in secondo luogo, fondare una solida tradizione storica, che comprovi le radici comuni del popolo africano. Nel primo caso, Asante entra in contraddizione non soltanto coi numerosi studi di sociologia e antropologia (come quelli di Balandier, Gluckman, Mitchell, Kuper e altri), da lui classificati come eurocentrici e, quindi, non attendibili, ma anche con posizioni espresse da altri eminenti pensatori africani o africanisti, come Franz Fanon. Quest'ultimo, ad esempio, aveva ribadito più volte la ricchezza e, quindi, l'eterogeneità presenti in seno al continente africano, rifiutando una concettualizzazione astrattamente unitaria ed essenzialista delle espressioni culturali africane. ${ }^{5} \mathrm{Nel}$ secondo, il riferimento essenziale è costituito dall'Antico Egitto. E qui Asante, sulla scia dei suoi illustri predecessori sopra ricordati, apre un dibattito che continua ancora oggi, in merito all'interpretazione delle fonti egizie che dovrebbero indicare le origini nere di tale civiltà, da cui avrebbe preso avvio il pensiero filosofico, scientifico e religioso. Asante sposa in pieno le teorie di Diop, Obenga e James, secondo cui le principali culture antiche, prima fra tutte quella greca, avrebbero attinto per sviluppare o, semplicemente, ripetere con scarse innovazioni ${ }^{6}$ il sapere contenuto nei Grandi Misteri Egizi, e trasmesso a pochi iniziati (fra cui Socrate).

In questo caso Asante utilizza il lavoro precedentemente fatto dai suoi predecessori, per dedicarsi maggiormente alla costruzione del nuovo paradigma in senso filosofico $\mathrm{e}$ ideologico, dando per acquisite e, quindi, valide, le conclusioni sulle fonti egizie proposte da Diop, Obenga, James e Bernal, con Black Athena (1987).

In questo articolo si cercherà di ricostruire il dibattito sulle fonti egizie sia storiche che filosofiche che, a partire dagli studi di Diop e James, si è venuto dipanando dagli anni Cinquanta a oggi.

I passaggi essenziali dell'operazione intellettuale promossa dall'Afrocentrismo sono fondamentalmente tre: in primo luogo, la dimostrazione che quella egizia è una civiltà nera, quindi africana; in secondo luogo, che da essa si siano diramate mediante la teoria del "diffusionismo", sia di tipo físico (migrazioni) che culturale (influenza diretta 0 indiretta), elaborata da Diop - quelle linee che avrebbero portato gli insegnamenti dei sacerdoti egizi su tutto il continente africano e

\footnotetext{
${ }^{5}$ F. FANON, Peau noire, masques blancs, 1952.

${ }^{6}$ G. JAMES, Stolen Legacy, 1954 (1989²).
} 
sul bacino euro-mediterraneo (Cartagine, mondo greco, ecc.); infine, l'idea del plagio, da parte dei Greci, nei confronti del sapere filosofico, scientifico e religioso originariamente prodotto dagli Egizi.

C'è un momento, nel dibattito storiografico, in cui queste due grandi tendenze vengaono a confronto, giungendo alla conclusione dell'impossibilità di qualsiasi tipo di conciliazione. Si tratta del Simposio del Cairo del 1974, organizzato dall'UNESCO, relativo alla ricerca di linee comuni per la scrittura della storia dell'Africa: emerge in questa occasione una tendenza minoritaria, capeggiata da Diop, definibile come «afro-centrica » (anche se Diop mai userà questo termine), che farà marcare tutto il suo dissenso rispetto a quella maggioritaria, cominciando a porre in modo consistente e sistematico le tesi che poi Asante assumerà in pieno con la formulazione dell'Afrocentrismo. In questo momento comincia a formarsi una scuola di pensiero tesa a $«$ inventare una tradizione $»,{ }^{7}$ mediante una rilettura radicale delle fonti storiche egizie, che lo stesso Asante, una trentina d'anni dopo, coglierà come essenziale per il percorso che porterà alla formulazione dell'Afrocentrismo: « Diop and Obenga - scrive Asante ${ }^{8}$ - walked toward fear and when they had finished presenting their papers they had shottered all of the lies that were told about Africans »".

Il presente articolo è composto da tre parti: nella prima si fornisce un breve inquadramento concettuale dell'Afrocentrismo; nella seconda si concentra l'attenzione sul dibattito intorno alle fonti storiche e filosofiche egizie, che l'Afrocentrismo porterebbe a prova delle sue teorie e dello Stolen legacy; infine, nell'ultima ci si concentrerà sulle alcune delle critiche ricevute dall'Afrocentrismo su tali questioni. Concluderà l'articolo una riflessione finale, sulla base delle considerazioni svolte durante il testo.

\section{L'Afrocentrismo: inquadramento storico-concettuale}

L'Afrocentrismo sorge in un clima politico e culturale ben identificabile: quello delle lotte degli afro-americani a partire dagli anni Sessanta fino agli anni Ottanta. Politiche di Affirmative Action, la fine della discriminazione razziale negli Stati Uniti, un nuovo spazio culturale e accademico per gli afroamericani (col primo programma dottorale in Studi Afroamericani sopra ricordato), insomma una tale, nuova atmosfera

\footnotetext{
${ }^{7}$ E. HobSBAWM \& T. RANGER (Eds.), The Invention of Tradition, 1983.

${ }^{8}$ M.K. AsANTE, « An African Origin of Philosophy: Myth or Reality? », 2004, ora in www.asante.net/articles/26/afrocentricity.
} 
rappresenta la base per la nascita di questa nuova corrente di pensiero. ${ }^{9}$

L'Afrocentrismo intende quindi rispondere «alla storica oppressione degli Afro-Americani », cercando di ricostruire «l'identità africana del popolo Africano ». ${ }^{10}$ Come Asante aveva evidenziato sin dalla sua opera inaugurale della corrente, il concetto di "popolo africano» è centrale per l'Afrocentrismo. ${ }^{11}$ I tre pilastri fondamentali su cui si erge l'edificio afrocentrico sarebbero allora i seguenti: una storia unica, segnata da tappe dolorose (colonialismo, schiavitù, segregazione negli Stati Uniti e in Sud Africa), ma anche da un inizio mitico, quello della civiltà egizia; uno stile cognitivo unico e peculiare, che rifiuta la suddivisione occidentale, di matrice aristotelica, di separazione mente/corpo, materia/anima; caratteristiche comuni delle lingue africane, a partire da quella dell'antico Egitto, e riscontrabili attraverso somiglianze di questa con alcuni idiomi africani contemporanei, como il wolof. ${ }^{12}$ Muovendo dalla necessità di costruire una «storia unica », Asante entra a pieno titolo in seno al dibattito sulle fonti storiche egizie, usando soprattutto gli studi di Diop e Obenga.

Ma l'Afrocentrismo non è una semplice rilettura del passato: piuttosto, esso tende a edificare un nuovo paradigma, proponendo una vera e propria teoria dell'agire di tipo politico e ideologico, addirittura profetico. Il popolo africano, composto sia dagli africani del continente che da quelli della diaspora, deve non soltanto ispirarsi ad un passato comune, ma anche intraprendere la strada verso un futuro di liberazione, lo « Njia » («cammino» in Swahili), al fine di raggiungere la piena maturità e coscienza della propria, comune identità collettiva, sintetizzabile col principio fondamentale del Maât, la GiustiziaLibertà. Un simile cammino passa per cinque fasi, culminanti nella piena auto-coscienza e auto-realizzazione, che significa l'abbandono delle categorie e della mentalità eurocentriche. L'Afrocentrismo non ammette identità multiple da parte dei suoi aderenti: l'unica, esclusiva identità deve essere quella di tipo etnico-razziale, escludendo così anche tutte le altre, ritenute concorrenziali e incompatibili, siano esse di tipo politico che religioso o filosofico o genericamente culturale $o$ anche sessuale.

\footnotetext{
${ }^{9}$ R. SINORITA FIERRO, L'identità afroamericana, 2001.

${ }^{10}$ R. SENTWALI BAKARI, «Epistemology from an Afrocentric Perspective $», 1997$.

${ }^{11}$ M. K. ASANTE, Afrocentricity, 1980.

${ }^{12}$ A. K. BANGURA, « From Diop to Asante », 2012.
} 
Nel prossimo punto cercheremo di presentare attraverso quali argomentazioni questa corrente interpreta la civiltà egizia come nera e africana, aprendo quindi la strada verso il percorso che porterà al nuovo paradigma asantiano.

\section{Le fonti storiche dell'Antico Egitto e la nascita dell'Afrocentrismo}

Le tesi «egittocentriche » dell'Afrocentrismo affondano le loro radici su una convinzione che Asante dà per scontata, ma di cui occorre chiarire i tratti essenziali: l'Egitto è una civiltà africana, nera, e da essa il genere umano, con le più evolute forme di civiltà, si è sparso verso il continente africano, parte dell'Europa e del Medio Oriente, mediante un processo di «diffusionismo », prima di tipo demografico, poi di carattere culturale. Quali sono le basi storiche che lo inducono a trarre simili conclusioni?

La risposta è fornita dalle ricerche sulla civiltà egizia di Diop e Obenga. È da qui che tutto l'Afrocentrismo si basa per affermare le sue tesi egittocentriche.

Le questioni essenziali sono le seguenti: 1. Che tipo di obiettivi hanno gli afrocentristi in riferimento all'interpretazione della civiltà egizia? 2. Sulla base di quali fonti tali obiettivi sono perseguiti? 3. Quali critiche tali percorsi «alternativi» hanno suscitato nel dibattito scientifico internazionale?

\subsection{Gli obiettivi}

L'Afrocentrismo necessitava, alla sua nascita, di una base storica solida, che fungesse da sostrato culturale comune per giustificare l'unità della civiltà africana. Una sola cultura antica poteva svolgere questo ruolo, quella egizia. Questa grande civiltà, relegata dal paradigma eurocentrico a una sorta di " premessa » della classicità greco-romana, non poteva che essere completamente nera.

Le circostanze storiche favoriscono l'approccio afrocentrico e egittocentrico di Diop, Obenga, James Bernal, Asante e altri almeno in due sensi: in primo luogo, vi era una lunga tradizione che oggettivamente aveva cercato di marginalizzare la civiltà egizia, classificandola come caucasica, cioè bianca, e quindi inferiore rispetto a quella greca. Non sarebbe stato difficile contrapporsi a una simile prospettiva, richiamando anche il razzismo insito in essa. Inoltre, le incertezze nel determinare il colore della pelle o la «razza » degli antichi Egizi (tema assai caro agli afrocentristi) continuavano a caratterizzare, negli anni Settanta e Ottanta, gli studi di antropologia fisica applicata all'Egitto (situazione protrattasi in larga parte fino a oggi, e di cui si dirà succintamente nel paragrafo 4). Se, quindi, il sistema ritenuto più valido per la determinazione della razza di un certo 
gruppo umano non poteva funzionare per gli antichi Egizi, era allora lecito utilizzare altre fonti e formulare ipotesi del tutto diverse rispetto a quelle per così dire « canoniche »o comunque più accreditate.

Nel prossimo punto si cercherà di chiarire che cosa gli afrocentristi, soprattutto per bocca di Diop, avevano riscontrato nel dibattito ottocentesco e primo-novecentesco in materia di teorie egittologiche, e come essi avessero trovato campo libero, vista l'impossibilità di stabilire con certezza il tipo umano a cui gli Egizi dovevano essere ascritti.

\subsection{L'identità razziale africana secondo la teoria afro- centrica}

Secondo Diop, vi sarebbero prove sufficienti per affermare che gli Egizi erano neri, e che la loro civiltà cominciò dall'Alto Egitto e non dal Delta del Nilo, ossia dalla parte certamente africana e nera e non da quella più prossima a influenze mediterranee. ${ }^{13}$ Varie fonti sono usate per corroborare una simile teoria: da Erodoto a Diodoro di Sicilia fino a Gaston Maspero. ${ }^{14}$ Tuttavia, ciò che appare maggiormente rilevante, nel tentativo di decostruzione del paradigma eurocentrico finalizzato a screditare la civiltà africana, è la tesi della « Moderna Falsificazione della Storia ». Essa avviene a partire dalla presunta inversione delle evidenze che Champollion aveva trovato, e che aveva trasmesso a suo fratello, ChampollionFigeac, attraverso una serie di lettere pubblicate nel 1833. Se le scoperte di Champollion costrinsero gli altri storici ad ammettere che quella egizia era la « più antica civiltà che generò tutte le altre $»,{ }^{15}$ l'imperialismo non poteva accettare che tale cultura fosse nera. Secondo Diop, fu a causa di queste esigenze di tipo puramente politico che prese avvio la falsificazione della storia. In primo luogo, egli considera il commento di Champollion al bassorilievo di Sesostri I (XVI secolo a.C.), in cui figurano le razze umane conosciute dagli Egizi. Nel lungo passo che Diop cita, Champollion afferma che Egizi e Africani sono rappresentati allo stesso modo, e chiaramente distinti in confronto ai Nampu (asiatici) e Tamhou (europei). Conclusione: gli Egizi erano neri, nonostante fossero rappresentati con un colore rossastro, probabilmente per motivi simbolici. ${ }^{16}$

Champollion-Figeac alterò significativamente le scoperte del fratello, arrivando ad affermare che il colore scuro della pelle e i

\footnotetext{
${ }^{13}$ C.A.Diop, The African Origin of Civilization, 1974, p.1.

${ }^{14}$ IDEM, p. 2.

${ }^{15}$ IDEM, p. 45.

${ }^{16}$ IDEM, p. 48.
} 
capelli lanuti non sono elementi sufficienti per riconoscere che gli Egizi erano neri, concludendo che fossero bianchi. ${ }^{17}$

Diversi autori condivideranno l'idea dell'ipotetica falsificazione della storia. Grosso modo negli stessi anni in cui, dopo la morte di Champollion, il fratello procede a una radicale reinterpretazione delle sue scoperte, negli Stati Uniti Samuel George Morton teorizza l'origine poligenetica di razze umane distinte, a cui il Signore aveva affidato funzioni e ruoli diversi e prestabiliti. Quello che da alcuni è stato considerato come il padre dell'antropologia fisica americana, dopo avere analizzato tre mummie egizie giunge alla conclusione che queste erano causasoidiche e non nere, e che la divisione fra razze doveva essere considerata come un dato originario, presente sin dagli albori dell'umanità. ${ }^{18}$ Tuttavia, in quegli anni le scuole di pensiero a proposito della razza degli Egizi erano ancora in disputa: se Morton, col suo saggio del 1844 ebbe una notevolissima influenza, le tesi opposte, sostenute da studiosi quali Blumembach e Prichard, avevano anch'esse una certa credibilità scientifica. ${ }^{19}$

L'ondata di positivismo che, verso la fine del XIX secolo e l'inizio del XX, attraversò grande parte della cultura occidentale, a partire da quella britannica, fece in modo che la tesi dell'Egitto come civiltà bianca o mediterranea prendesse il sopravvento. Illustri personaggi quali Elliot Smith, pluridecorato dalla Royal Society e dal Royal Anthropological Institute of Great Britain and Ireland, e Arthur Keith difesero la teoria dell' « iperdiffusionismo », secondo cui l'origine della civiltà va ricercata nella razza bianca o caucasica, e che l'Egitto faceva parte a pieno titolo di tale razza. Da qui si sarebbero diffusi tutti quei segni di civiltà che avrebbero poi portato all'affermazione delle più elevate manifestazioni umane nell'antichità. ${ }^{20}$ Idee non dissimili proposero il Giuffrida-Ruggeri, che riteneva che gli abitanti dell'Alto Egitto fossero Etiopici, e quelli del Basso bianchi mediterranei, e soprattutto il Coon che, nel 1939, scrisse un testo che avrebbe destato scalpore, a causa delle sue tesi apertamente razziste, sostenendo - da posizioni ancora una volta poligenetiche - che la razza nera avrebbe avuto un ritardo, nella scala evolutiva, di circa 200.000 anni rispetto a quella bianca. ${ }^{21}$

\footnotetext{
${ }^{17}$ IDEM, p. 51.

${ }^{18}$ S.G. Morton, Crania Aegyptiaca, 1844.

${ }^{19}$ Cf., su questo dibattito, C.RIGGS, Unwrapping Ancient Egypt, 2014.

${ }^{20} \mathrm{Cf}$, , fra i più recenti studi su questa figura, P.CROOK, Grafton Elliot Smith, Egyptology \& the Diffusion of Culture, 2012.

${ }^{21}$ C.S. CoOn, The Race of Europe, 1939 e C.S. CoON, The Origin of Races, 1962.
} 
Diop avvia la sua riflessione e la sua produzione scientifica sull'Egitto cercando di ribattere a simili posizioni, secondo un programma apertamente ideologico. Come è stato rilevato, il sospetto che le sue tesi difendano un « razzismo al contrario » è piuttosto forte. ${ }^{22}$ Diop, dunque, cerca di ribaltare completamente l'apparato ideologico che mirava a svalutare i neri africani. Dimostrando le origini africane e nere dell'antico Egitto, Diop avrebbe avuto buon gioco in un senso molto più lato, affermando cioè la superiorità della razza nera, da cui si sarebbe diffusa la civiltà verso l'Europa, il Medio Oriente e il resto del continente africano.

In che modo Diop cerca di decostruire le ipotesi poligeniste, anti-africaniste e che vedevano l'Egitto come civiltà debitrice della razza caucasica?

Fondamentalmente attraverso due tipi di prove: una, per così dire diretta, maggiormente orientata verso ritrovamenti archeologici e, quindi, l'antropologia fisica, l'altra indiretta, incentrata su aspetti demografici e culturali e linguistici, su cui lo studioso senegalese insisterà di più, e che finirà col prevalere nel movimento afrocentrista contemporaneo.

Diop analizza con molta attenzione le varie ipotesi relative all'origine degli antichi Egizi: egli considera l'origine locale, suddivisa fra Basso e Alto Egitto, e l'origine asiatica. Nel primo caso, la teoria che insiste nella «preponderanza del Delta », ${ }^{23}$ sostenuta, fra gli altri, da Alexandre Moret, sottolinea la caratteristica bianca, caucausica, dei nativi. Ma Diop rileva come evidenze archeologiche dimostrino che fu a partire dall'Alto Egitto che prime forme di civiltà cominciarono a formarsi, dando gli esempi di Tasian, Badarian, Amratian (quest'ultima verso il 6.500 a.C.). Nel Delta (Basso Egitto) tali forme fecero la loro comparsa più tardi. Inoltre, come prova indiretta, Diop cita il fatto che gli Egizi mai diventarono una grande potenza marittima, e che chiamassero il mare una "secrezione contaminata", secondo quanto scritto da Plutarco. ${ }^{24}$ Ciò significherebbe che l'origine di questa civiltà a partire dal Delta sarebbe impossibile.

Anche l'ipotesi di un'origine asiatica degli Egizi è smentita da Diop. La maggior prova che lo induce a scartare una simile idea è, ancora una volta, di tipo indiretto: Diop deduce che una qualsiasi origine esterna della civiltà egizia presupporrebbe l'esistenza di un'altra precedente e più sviluppata, capace di

\footnotetext{
${ }^{22}$ Espressione usata da C.L. Brace, D.P. Tracer et Alit, «Clinn and Clusters Versus ‘Race'», 1993.

${ }^{23}$ C.A. Diop, The African Origin of Civilazation, 1974, p. 84.

${ }^{24}$ IDEM, p. 98.
} 
colonizzare il territorio egizio. Circostanza che, anche a opinione di famosi egittologi, non sembra confermata. ${ }^{25}$

Mancando evidenze scientifiche certe che confermino la natura nera della civiltà egizia, Diop ricerca quindi elementi indiretti. Questi lo aiuteranno a formulare l'idea dell'esistenza di una comume civiltà africana fra Egizi e il resto degli Africani, alimentando il mito dell'unità culturale del continente. Come lo stesso Diop scrisse nella prefazione di un suo famoso testo, l'obiettivo di gran parte dei suoi studi è « a true reinforcement of our feeling of cultural oneness $\gg{ }^{26}$

A partire da un tale programma ideologico assai più che scientifico, Diop comincia con l'evocare una serie di pratiche culturali comuni a tutto il territorio africano. Il totemismo, ad esempio, assente fra le popolazioni bianche, è tipico di quelle nere, fra cui quella egizia, così come la circoncisione, diffusa fra gli Egizi sin dai tempi preistorici. Citando i lavori di Griaule sui Dogon, Diop sostiene che l'unica forma di circoncisione coerente con una certa visione dell'universo e del cosmo è quella degli Egizi: infatti, essa deve essere accompagnata dalla escissione, secondo una logica in cui è necessario ritirare qualcosa di femminile all'uomo e di maschile alla donna, al fine di rendere evidente il sesso del neonato che, a priori, è in certo senso androgino. ${ }^{27}$ Così, Diop formula l'ipotesi secondo cui l'androginia divina deve essere esistita anche nella società egizia. Il terzo elemento evidenziato è il concetto della Kingship; la «nobiltà reale » è caratteristica dei popoli africani, compresi gli Egizi, essenzialmente attraverso una pratica specifica: la condanna a morte del monarca una volta superata una certa età, a causa della perdita della salute e, con essa, della sua forza ed energia che gli permettono di governare. Diop osserva che questa pratica, che nasconde una visione vitalista dell'essere umano e in particolare dell'essenza della nobiltà reale, è presente in Egitto così come entro i Mbum dell'Africa Centrale, gli Yoruba, i Dagomba, gli Shamba, gli Igara, ecc. ${ }^{28}$ Il quarto elemento è la cosmogonia. Diop cita il classico testo di Temples, Filosofia Bantu, scritto nel 1959, per mostrare le somiglianze impressionanti fra le concezioni del cosmo degli Egizi e della maggioranza delle tribù africane anche contemporanee. Quindi, la base della filosofia egizia dovrebbe essere ricercata in una mentalità tipicamente africana. Anche la stratificazione sociale confermerebbe, a detta di Diop, la

\footnotetext{
${ }^{25}$ A. Moret \& G. DAVy, From Tribe to Empire, 1970, pp. 132-133.

${ }^{26}$ C.A. DIOP, Precolonial Black Africa, 1987, p. XII.

${ }^{27}$ C.A. Diop, The African Origin of Civilization, 1974, p. 136.

${ }^{28}$ IDEM, p. 139.
} 
comunanza fra Egizi e tutti gli altri africani, essendo presenti, nell'un caso e nell'altro, le seguenti classi (anzi, caste): contadini, artigiani, sacerdoti, guerrieri e ufficiali di governo, infine il re. Diop ritiene che il sistema europeo sia caratterizzato da vere e proprie classi sociali, mentre quello africano (ed egizio) da caste, in cui, nonostante l'impossibilità di mobilità sociale verso il basso e verso l'alto dei suoi membri, vi sarebbe maggiore omogeneità e solidarietà, in ragione del fatto che tutti, in un modo o nell'altro, compartecipano al potere reale e che gli stessi schiavi non avrebbero subito quello sfruttamento intensivo da parte dei signori, tipico del Medioevo occidentale. I gér, la casta superiore in Africa, " could not materially exploit the lower classes without losing face in the eyes of others, as well as their own $\gg{ }^{29}$ Anticipando una delle critiche rivolte al movimento afrocentrista, Howe ricorda, polemicamente, che la tendenza a negare, contro ogni evidenza, l'esistenza della schiavatù nell'Africa pre-coloniale, al massimo riducendola a un fenomeno marginale, tutto sommato benigno, rappresenta una pura fantasia. ${ }^{30}$ Nel Sudan Centrale e nella città-stato di Hansa la percentuale di schiavi oscillava fra il 30 e $50 \%$ della popolazione locale, nel regno Fulani fra il 30 e il $66 \%$, nella zona del Senegal, Gambia e Sierra Lene da un minimo di 30 a un massimo del $75 \% .^{31}$ La stessa società egizia era certamente basata sulla manodopera schiavista. Un noto ricercatore africano definisce la società egizia come fortemente gerarchica, del tutto distante da quegli ideali di eguaglianza, libertà, rispetto per $\mathrm{i}$ diritti dell'individuo, che il movimento afrocentrista (classificato come « flok-philosophy ») invece sostiene. ${ }^{32}$

Da ultimo, la lingua. Secondo Diop, sarebbe facile provare la profonda unità fra lingua egizia e lingue africane moderne, così come diffícile sarebbe trovare somiglianze fra l'idioma dell'antico Egitto e quelli di origine indo-europea e semita. Lo wolof, lingua madre di Diop, mostra evidenti vicinanze con l'egiziano antico. Conclusione: si tratta di gruppi linguistici con una stessa origine e tratti comuni, evidenti sino ai nostri giorni.

\footnotetext{
${ }^{29}$ C.A. Diop, Precolonial Black Africa, 1987, p. 2.

${ }^{30}$ S. Howe, Afrocentrism, 1988, p. 149.

${ }^{31}$ T.C. PATterson, Making Alternative Histories, 1982, pp. 354-356.

${ }^{32}$ K.A. AppiaH, In My Father's House: Africa in the Philosophy of Culture, 1992, p. 207.
} 


\subsection{Il Simposio del Cairo: due scuole a confronto, nessuna convergenza}

In occasione del Simposio del Cairo organizzato dall'UNESCO nel 1974 le posizioni di Diop (e Obenga) si scontrano con quelle per così dire ufficiali.

L'incontro s'intitola The peopling of ancient Egypt and the deciphering of Meroitic script, e l'obiettivo principale è sviluppare « a faithful reflection of the way in which African authors view their own civilization $» .{ }^{33}$ Probabilmente, vi era un fraintendimento che stava alla base dell'intera filosofia dell'opera: «Africa will be considered as a totality ». ${ }^{34}$ Indirettamente, un simile approccio favoriva le teorie afrocentriche e egittocentriche degli afrocentristi, senza averne dato preventivamente una giustificazione di carattere scientifico e storico razionale.

Il rappresentante della teoria maggioritaria era Jean Vercoutter, che si scontrò a più riprese con Diop e Obenga. Il punto di divergenza maggiore era legato ai risultati, ma, probabilmente, ancora più rilevante è la questione metodologica, relativa alle fonti usate e alla loro gerarchia. Vercoutter riteneva che le fonti disponibili per determinare la natura fisica e culturale della civiltà egizia dovessero essere le seguenti (in ordine di importanza e attendibilità): 1. Scientifiche in senso stretto (antropologia fisica); 2. Iconografiche; 3. Linguistiche; 4. Etnologiche.

Al contrario, Obenga teorizzò che siano le fonti che Vercoutter considerava come meno rilevanti a occupare il primato al fine di stabilire la natura della cultura e della razza degli antichi Egizi. Il suo argomento affondava le radici nell'insegnamento di De Saussure, secondo il quale il legame linguistico sarebbe essenziale per rivelare relazioni certe fra culture diverse. L'obiettivo, quindi, era stabilire rapporti linguistici (fonetici, semantici, morfologici, ecc.) fra l'antica lingua egizia e le moderne lingue africane. Il fatto che il suffisso $-t$, che esprime il femminile nell'antico egiziano, abbia la stessa funzione in molte lingue africane moderne costituirebbe già una prova di tale legame culturale. ${ }^{35}$ D'altra parte, il suo maestro Anta Diop aveva già sperimentato tale metodologia studiando le somiglianze fra l'antico idioma egizio e il wolof.

${ }^{33}$ UNESCO, Foreword: Preparation of a General History of Africa, 1978, p. 9.

${ }^{34}$ IDEM, p. 8.

35 T. OBENGA, «The genetic linguistic relationship between Egyptian (ancient Egyptian and Coptic) and modern Negro-African language », 1978. 
Oltre alla metodologia, anche gli obiettivi di questi due gruppi di studiosi appaiono dissimili: da un lato, si ricercano affinità culturali, cercando di spiegare essenzialmente mediante le evidenze dell'antropologia fisica la formazione dello Stato egizio; dall'altra, gli afrocentrici cercano di spostare la discussione su un unico punto: la razza degli antichi Egizi.

Il consenso non si trova. Leggendo i tentativi di sintesi che, su ogni aspetto del simposio del Cairo, vengono via via elaborati, le distanze si presentano come incolmabili. Prendiamo alcuni esempi. Rispetto alla questione relativa al colore della pelle degli antichi egizi, si può leggere: « It was not possible to take the discussion in this field and the symposium was unable to make any clear recommendation on the question at this point of the debate $\gg{ }^{36}$ Conseguentemente, anche sulla questione della formazione dello Stato egizio (per migrazioni dall'Asia o grazie a forze endogene?) non c'è alcun accordo. Sulla composizione fisica della popolazione dell'antico Egitto le posizioni che emergono sono ancora opposte, oscillando fra l'idea di un gruppo omogeneo e di uno «misto ». ${ }^{37}$ Infine, il colpo finale a un possibile consenso è portato da Diop, il quale cerca di smentire l'idea che le fonti più attendibili siano quelle dell'antropologia fisica, profilando cosí una sorta di agenda di lavoro autonoma della corrente afrocentrica non soltanto in relazione alla scrittura della storia dell'Africa sotto l'egida dell'UNESCO, ma anche per il futuro: «Finally, Prof. Diop mentioned that racial type could not at present be established on the basis of a craniometric evidence alone but that conclusions could be arrived at if this evidence alone were combined with osteological data $\gg .{ }^{38}$ Neanche per quel che riguarda la parte iconografica fu raggiunto un accordo, cosa che avvenne, in parte, soltanto per gli aspetti linguistici.

\section{Dopo il Simposio del Cairo: il consolidamento del pensiero afrocentrico dal "diffusionismo" allo "Stolen Legacy"}

Il Simposio del Cairo è stato un momento decisivo per prendere atto, da parte della comunità scientifica internazionale, dell'esistenza di due scuole di pensiero: una, sempre più protesa a cercare di comprendere l'effettiva natura, física e culturale, degli abitanti dell'antico Egitto, l'altra, quella afrocentrica, che ha dato ormai per acquisite e valide gran parte delle intuizioni di

\footnotetext{
${ }^{36}$ UNESCO, Symposium on the Peopling of Ancient Egypt, A Report on the discussion, 1978, p. 88.

${ }^{37}$ IDEM, p. 94.

${ }^{38}$ IDEM, p. 98.
} 
Diop e del suo allievo, Obenga, fondando una teoria filosofica e una vera e propria ideologia su di esse.

L'Afrocentrismo di Asante compie, però, un passo ulteriore: col decisivo contributo indiretto di James, e diretto di Obenga, non soltanto si limita a formulare una teoria egittocentrica e unitaria della civiltà africana, ma giunge a riesumare $\mathrm{e}$ corroborare la tesi dello "stolen legacy », ossia dell'eredità rubata. Il tutto ha molto a che vedere con la filosofia, la scienza, la religione e laloro ipotetica origine in Egitto.

In questo punto si cercherà di mostrare, illustrando l'uso delle fonti storiche ma soprattutto di quelle filosofiche e scientifiche, come tale formulazione sia sorta e come abbia trovato sempre maggiori consensi all'interno del movimento afrocentrista e sempre più aspre critiche al di fuori di esso, soprattutto da parte degli studiosi del mondo egizio e di quello classico.

Tutto ha inizio col diffusionismo, che Diop aveva utilizzato per spiegare l'unità culturale e fisica africana, e che adesso il movimento afrocenstrista ripropone sino a toccare il contesto europeo e mediterraneo. Vi sono due modi per leggere il diffusionismo: in prima istanza, interpretare il fenomeno dal punto di vista puramente demografico; quindi, e conseguentemente, in senso culturale.

Secondo Diop, grandi migrazioni dall'Africa Orientale verso il resto del continente avrebbero caratterizzato l'espansione egizia, una prima volta circa 3 milioni di anni fa, una seconda circa 200.000 .

Successivamente a Diop, altri studiosi recuperano e sviluppano l'argomento. Il più noto e influente è Martin Bernal, secondo il quale ci fu un'influenza diretta e significativa degli Egizi nella cultura greca, mediante un'invasione militare. ${ }^{39}$ All'inizio del II millennio a.C., Senwosret I e III avrebbero fatto campagne belliche fra Europa e Vicino Oriente, invadendo Anatolia, Bulgaria e Grecia. Esponenti delle ali più radicali del movimento afrocentrista e rastafari credono che questo grande re egizio abbia fondato Atene. ${ }^{40}$

Questa antica invasione rappresenterebbe il primo elemento che comproverebbe che ci sai stato, in effetti, un contatto diretto e prolungato fra Egizi e Greci, e che quindi i primi avrebbero trasmesso sin da questa remota epoca nozioni e concetti fondamentali di scienza e filosofia al popolo ellenico.

\footnotetext{
${ }^{39}$ M. BERNAL, Black Athena, 1987, p. 568.

${ }^{40}$ Per questa interpretazione, cfr. S.D. EWING, Sesostris the Great, the Egyptian Hercules, 2006.
} 
La seconda circostanza per spiegare la grande influenza egizia e, pertanto, nera in rapporto alla cultura greca è data dalle teorie dei principali filosofi greci: si tratta di un metodo estremamente indiretto per comprovare un rapporto tanto decisivo, ma che per gli afrocentristi rappresenta uno dei più affidabili. In questo caso, l'analisi del pensiero filosofico e scientifico è spalleggiata dalle testimonianze di epoca greca, in primo luogo Erodoto e Diodoro Siculo, ma anche gli stessi filosofi, fra cui Platone.

L'idea fondamentale era stata espressa da George James, già intorno agli anni Cinquanta: tutta la filosofia greca, dai presocratici fino a Socrate passando per Platone e Aristotele, niente altro sarebbe se non una derivazione e ripetizione dei Misteri egizi. ${ }^{41}$ L'influenza egizia non si limitava, naturalmente, al mondo greco: anche le scuole filosofiche caldee e, in un'epoca successiva, romane, mostravano una chiara impronta kemetica. Quali elementi apporta James per provare tale affermazione?

E possibile riassumere gli argomenti di James in quattro punti: 1. Le condizioni politiche greche avrebbero reso impossibile l'elaborazione di un pensiero filosofico autonomo e così sviluppato, come la tradizione occidentale ha lasciato credere. Dal tempo di Talete a quello di Aristotele la Grecia fu costantemente sottoposta a divisioni intestine e addirittura guerre, che non avrebbero lasciato il tempo affinché scuole di pensiero tanto complesse potessero sorgere. Nei periodi di relativa pace interna, la minaccia persiana costituiva un ulteriore motivo di grande preoccupazione. Così conclude James: « ... it is an accepted truth that the development of philosophical thought requires an environment which is free from disturbance and worries. The period commonly assigned to Greek philosophy (i.e. Thales to Aristotle) was exactly the opposite to one of peace and tranquility, and therefore it could not be expected to produce philosophy $\gg .{ }^{42}$ A riprova di ciò, James ritiene che una figura come Aristotele si posizionasse a una distanza temporale di circa 5000 anni dal contesto in cui viveva, ragion per cui se ne può dedurre che « it is impossible to escape the conviction that he obtained his education and books from a nation outside of Greece ». ${ }^{43}$ E l'unica nazione possibile per esercitare tale influenza era 1'Egitto; 2. La filosofia greca era in larga misura la ripetizione di quella egizia, come dimostrerebbero alcuni elementi comuni (o pressoché identici) alle due scuole: primo fra tutti, la teoria della salvezza, principale preoccupazione del pensiero egizio, ripresa da autori

\footnotetext{
${ }^{41}$ G. JAMES, Stolen Legacy, $1954\left(1989^{2}\right)$.

${ }^{42}$ IDEM, p. 26.

${ }^{43}$ IDEM., p. 120.
} 
quali Platone e Plotino, e che includerebbe l'arte, la musica, le arti liberali, la matematica, insomma una serie di discipline che, lette mediante un'ottica olistica, farebbero propendere per un plagio operato dai filosofi greci a danno di quelli egizi. Socrate, che sarebbe stato un iniziato diretto dei grandi sacerdoti egizi, avrebbe da loro preso la sua filosofia basata sul «conosci te stesso ॥; la dottrina etica di Platone apparterrebbe integralmente al sistema dei Misteri egizi, mentre anche il Dio «motore immobile » di Aristotele « is non other than the Atum of the Memphite Theology of the Egyptians, the Demiurge $\gg .{ }^{44}$ Anche la dottrina atomistica di Democrito e quella degli opposti di Eraclito sarebbero state imparate dai rispettivi autori in Egitto; ${ }^{45}$ 3. Stesso discorso vale per altri ambiti filosofici, quali l'etica, in cui il controllo dell'azione e del pensiero, l'assenza del risentimento e della vendetta, l'ideale della fedeltà, sono tutti aspetti tipici del pensiero egizio, che sarebbero poi passati in quello greco; 4. In Egitto operava una grande Loggia, comandata dai sacerdoti, che aveva associato scuole filosofiche in tutto il mondo antico, fra cui quella greca. James ritiene che il fatto che il Tempio di Delfi sia stato ricostruito nel 548 a.C. dal faraone Amasis indica l'enorme interesse degli Egizi rispetto a tutto ciò che aveva a che fare col mantenimento della loro egemonia intellettuale nelle aree limitrofe alla madrepatria. A questi aspetti ne va aggiunto un altro: praticamente tutti i filosofi greci, dai pre-socratici ad Aristotele, avevano conosciuto direttamente l'Egitto, poiché lì si erano recati al fine di imparare dai sacerdoti i misteri della loro dottrina, per poi rielaborarli e riproporli in chiave leggermente diversa. Secondo James, filosofi quali Pitagora, Talete, Anassimandro, Anassimene, Xenofane, Parmenide, Zenone, Melisso, Eraclito, Empedocle, Anassagora, Democrito, oltre a Socrate, Platone e Aristotele, tutti questi avrebbero ricevuto un insegnamento diretto in Egitto da parte dei sacerdoti, e ne sarebbero rimasti enormemente colpiti e influenzati. Questi argomenti spiegherebbero, a detta di James, lo Stolen Legacy e, quindi, il plagio esercitato dai Gregi verso gli Egizi.

L'altro autore che cerca di dimostrare, soprattutto mediante inferenze di tipo linguistico e filosofico, il rapporto gerarchico fra Egizi e Greci, da un punto di vista intellettuale e culturale, è Obenga. Le sue argomentazioni vengono sviluppate mediante un'analisi piuttosto accurata del pensiero filosofico egizio in rapporto a quello greco. Il metodo, però, è grosso modo lo stesso di quello di James: la presunzione che somiglianza di pensiero significhi derivazione, consequenzialità. In questo caso, gli aspetti centrali di carattere filosofico su cui Obenga insiste per

\footnotetext{
${ }^{44}$ IDEM, p. 118.

${ }^{45}$ IDEM, pp. 75-76.
} 
procedere alla sua dimostrazione sono essenzialmente tre: 1 . La filosofia nasce in Egitto perché gli Egizi avevano posto, assai prima dei Greci, gli interrogativi tipici di un pensiero speculativo astratto. Essi, infatti, ad esempio nel Testo delle Piramidi, risalente a oltre 2500 anni prima di Cristo, avevano cercato di interrogarsi sull'origine del cosmo, dell'uomo e sulle possibilità di conoscenza che questi aveva rispetto al mondo della natura. A questo proposito, gli Egizi avevano postulato l'esistenza del $N n w$, il Nous, principio unificatore, l' « elemento primordiale ", l' «acqua abissale » antecedente alla stessa nascita dei faraoni e alla stessa materia, paragonato da Obenga alla ragione spermatica degli Stoici, e prossimo alle prime elaborazioni dei « naturalisti » greci, come Talete. ${ }^{46}$ L'idea di un mondo costruito mediante una concezione meccanicista, « un congiunto architettonico impressionante $»,{ }^{47}$ risale anch'essa agli Egizi. Tale principio, secondo Obenga, è superiore rispetto all'idea platonica del Demiurgo, poiché quest'ultimo preesiste alla nascita del mondo, alla stessa stregua di ciò che avviene per il sumero Enki e per il cristiano Elohim. ${ }^{48}$ La conclusione, quindi, è che «bien avant le philosophe grec [Platon], les ancient Egyptiens ont conçu, à l'origine du monde, une matière antérieure au monde, incréée mais 'apte' à devenir la matière de la création $» ;{ }^{49}$ 2. Gli elementi primordiali, tipici della prima grande stagione filosofica greca, sarebbero stati scoperti e rielaborati molto tempo prima degli Egizi. Obenga cita ancora una volta il Testo delle Piramidi ( $\$ 2063 \mathrm{a}-\mathrm{b})$ per comprovare tale affermazione; 3 . In ambito etico, i principi poi proposti soprattutto dalla scuola platonica e dai suoi discepoli, fra cui, in parte, lo stesso Aristotele, la gerarchia e l'ordine cronologico fra Egizi e Greci sarebbero evidenti. Citando uno studio del $1956 \mathrm{su}$ Ptahhotep, uno dei filosofi etici egizi di maggiore rilievo, ${ }^{50}$ il cui pensiero verrà meglio sviluppato da Asante, Obenga riferisce che il suo più significativo insegnamento, il $T p-H s b, \mathrm{o}$ «metodo", retta norma, si ritrova esattamente in Platone.$^{51} \mathrm{~L}$ 'ordine morale varrebbe dunque come imperativo categorico, e sfocerebbe nel Maât, il principio supremo di Giustizia-Verità, foriero di pace e nemico del crimine; 3. Dal punto di vista scientifico, Obenga ricorda come le costruzioni delle grandi piramidi sottindendevano un livello di conoscenza

\footnotetext{
${ }^{46}$ L. BUSSOTTI, Afrocentrismo e questione religiosa, 2010, p. 139.

${ }^{47}$ T. OBENGA, La philosophie africaine de la période pharaonique. 2780330 avant notre ère, 1990, p. 36.

${ }^{48}$ IDEM, pp. 30-31.

${ }^{49}$ IDEM, p. 31.

${ }^{50}$ Z. ŽÁBA, Les Maximes de Ptahhotep, 1956.

${ }^{51}$ T. OBENGA, La Philosophie africaine, p. 156.
} 
di tipo matematico e ingegneristico che poteva derivare esclusivamente dalla consapevolezza di forme di pensiero astratto, teoretico. Lo stesso Aristotele, sostiene Obenga, in Metafisica, A. 1, 981 b 23, afferma che le matematiche sono di origine egiziana; le nozioni che gli Egizi avevano in ambito medico, cartografico, astronomico costituivano un patrimonio non soltanto di tipo meramente tecnico, ma scientifico nel senso più proprio del termine.

Nella stessa sfera religiosa, il monoteismo avrebbe inizio a partire dall'antico Egitto. Ciò sarebbe evidente nell'esperienza di Mosè, che è africano, e la cui lingua (l'ebraico) ha origini africane; per non parlare, poi, dei costumi che egli trasmise al popolo giudaico, come la circoncisione maschile, il tribalismo, la poligamia. $^{52}$ Anche in riferimento al Cristianesimo, gli afrocentristi ne difendono l'origine africana: ciò è dovuto, da un lato, a motivi «indiretti » (a causa della sua origine ebraica), dall'altro a motivi «diretti », ad esempio il fatto che Gesù abbia vissuto, durante i suoi primi cinque anni di vita, in Egitto; oltre a ciò, vari dei fondatori del pensiero cristiano sono africani, come Agostino d'Ippona e altri, che furono perfino eletti papi.

Dinanzi a un quadro già piuttosto complesso, Asante non fa che prendere spunto e approfondire alcuni degli elementi che alcuni dei suoi predecessori avevano elaborato, dedicandosi ad analizzare ulteriormente le implicazioni della filosofia egizia dinanzi al suo progetto, volto a costruire il nuovo paradigma afrocentrico. Ormai gli appare chiaro che una nuova cosmologia, epistemologia, etica, estetica possono essere fondate a partire dalla filosofia egizia, di matrice africana. Kemet è perciò l'inizio della conoscenza, ${ }^{53}$ la sua teoria afrocentrica il culmine di un lunghissimo percorso che adesso può vantare illustri riferimenti. Nelle note che egli dedica alla dimostrazione della derivazione egiziana della filosofia greca, Asante coglie essenzialmente due elementi che valgono la pena di essere qui ricordati: in primo luogo, il termine stesso « filosofia » rivela un'etimologia tutta egiziana. Infatti, secondo Asante, «Sofia » deriverebbe, in prima istanza, dalla parola egizia Mdu Ntr, ossia Seba, il saggio, trovata per la prima volta nel 2052 a.C., presso la tomba di Antef I. da cui si è originato il termine Saba (copto) e Sofia (greco). In secondo luogo, la dettagliata analisi di tutto il pensiero etico egizio, esaminando quelli che, a sua opinione, sarebbero i principali moralisti quali Imhotep, Ptahhotep, Akhenaten, Khumanup, i cui sistemi starebbero alla base dell'etica di pensatori quali Platone e

\footnotetext{
${ }^{52}$ Cf., su questo, S.B. NJAMI-NwADI, « Origine Africaine des principales religions révélées », 1993.

${ }^{53}$ Cf. M.K. AsAnTE, Kemet, Afrocentricity and Knowledge, 1990.
} 
Aristotele. Per giungere alla seguente conclusione: «Philosophy begins first with the black skinned people of the Nile Valley around $2800 \mathrm{BC}$, that is, 220 years before the apparence of Thales of Miletus $\gg{ }^{54}$

Nel prossimo punto vedremo in che modo i critici di questa teoria sono intervenuti per contraddirla e metterne in crisi i suoi presupposti fondamentali. Ancora una volta, partendo dalla sua base egiziana.

\section{Le critiche all'Afrocentrismo}

Le principali critiche che vengono mosse alla corrente afrocentrica si sono concentrate sulla solidità della costruzione del suo edificio teorico soprattutto da un punto di vista della validità storica, assai più che sotto il profilo squisitamente filosofico. In estrema sintesi, è possibile ricordare le seguenti:

- Questioni relative alla razza degli Egizi;

- Influenza degli Egizi rispetto alla formazione del «popolo africano », nel continente e nella diaspora (tesi dell' « unanimismo ») ${ }^{55}$;

- Questioni inerenti al ruolo degli africani (specialmente degli Egizi) in relazione ai vari ambiti del sapere, soprattutto per quel che concerne i suoi rapporti col mondo greco e, pertanto occidentale (lo «stolen legacy »).

\subsection{Questioni relative alla razza degli Egizi}

Nonostante Asante e i suoi colleghi ritengano acquisito il dato degli Egizi come popolo nero e, quindi, africano, le prove che Diop aveva raccolto sono tutt'altro che definitive per chiudere la questione. E proprio da qui cominciano le critiche degli storici verso l'Afrocentrismo.

Ricordato che la preoccupazione per la razza e il colore della pelle è eminentemente moderna, il confronto fra gli afrocentristi e gli storici dell'antichità su questo tema è assai intenso.

Uno degli aspetti che Diop, nel suo libro, aveva evidenziato era costituito da immagini di bassorilievi, pitture, monumenti che l'autore non commenta neanche, poiché sarebbero autoevidenti, mostrando come quello egizio fosse un popolo nero. Ma questa opinione non è condivisa da molti storici contemporanei.

\footnotetext{
2004.

${ }^{54}$ M.K. AsAnte, « An African Origin of Philosophy: Myth or Reality? »,

${ }^{55}$ S. Howe, Afrocentrism. Mythical Pasts and Imagined Homes, 1998, p. 2 .
} 
Uno di questi, ad esempio, ritiene che l'analisi dei crani degli Egizi dimostra che "gli antichi Egizi non erano né 'negri' né 'bianchi', ma Egizi, una popolazione dalle origini in maggioranza indigene e con un alto livello di continuità nel tempo $\gg .{ }^{56}$ Perfino autori africani riconoscono tale circostanza, usando le pitture egizie che sono arrivate fino a noi. ${ }^{57}$

Nei loro autoritratti, gli Egizi stilizzano costantemente le figure, in modo che queste risultino di difficile interpretazione: alcune hanno i capelli rossi, altre neri. Gli uomini sono generalmente più scuri, le donne più chiare, e gli schiavi sono rappresentati con la pelle scura.

Gli afrocentristi danno una lettura simbolica a tale diversità pittorica: a loro opinione, il vero colore della pelle (e dei capelli) sarebbe nero, raffigurando perciò individui africani. Al contrario, $\mathrm{i}$ critici vedono in un simile guazzabuglio la prova che il colore scuro fosse disprezzato dagli Egizi, tanto che le donne erano dipinte a tinte più chiare rispetto agli uomini e, soprattutto, gli schiavi avevano sempre una tonalità della pelle molto scura. Quest'ultima circostanza suggerisce che essi possano essere Nubi, in rapporto ai quali gli Egizi ebbero solitamente un atteggiamento di superiorità, visto che furono quasi sempre vicini-nemici e molti di loro furono catturati e ridotti in schiavitù. È quindi probabile che essi fossero, in effetti, più scuri, associando tale colore della pelle al disprezzo per $\mathrm{i}$ nemici. Nei rari momenti in cui i Nubi si allearono con gli Egizi, le rappresentazioni pittoriche cambiano, venendo i primi rappresentati con un colore della pelle più chiaro, assai prossimo a quello degli Egizi: ciò confermerebbe che, in queste epoche, si intendesse rappresentare $i$ due popoli come se si trattasse di uno solo, senza distinzioni fisiche e culturali. ${ }^{58}$ In generale, l'arte egizia aveva la tendenza «di rappresentare l'Egitto come completamente isolato da e superiore a tutti i suoi vicini Africani e di disegnare le terre del sud - Yam, Kush, Punt come meri strumenti di tributi esotici o obiettivo di conquista $\gg .{ }^{59}$

Anche le fonti letterarie rivelano un elevato grado di ambiguità e incertezza esegetica, poiché gli scrittori egizi non menzionano caratteristiche razziali, e quindi gli afrocentristi possono far ricorso esclusivamente a fonti posteriori e indirette, greche e romane. Queste sono solite distinguere fra «Etiopici» (più scuri) ed «Egizi» (più chiari). Pertanto è scorretto

\footnotetext{
${ }^{56}$ IDEM, p. 131.

${ }^{57}$ M. KEYTA, Race and the Writing of History, 2000.

${ }^{58}$ Cf. W. Adams, Nubia: Corridor to Africa, 1977.

${ }^{59}$ M. VAN WICK SMith, The First Ethiopians, 2009, p. 126.
} 
identificare i primi coi secondi, visto che le fonti classiche li distinguono chiaramente, ponendo in crisi l'intero edificio afrocentrico. $^{60}$ Inoltre, la costante attenzione nel voler valorizzazione la civiltà egizia fa in modo che gli afrocentristi disprerzzino, di fatto, un'altra grande civiltà, questa sì pienamente « nera », quella della Nubia, a sud dell'Egitto.

Una delle principali fonti utilizzate da parte degli afrocentristi (soprattutto Diop) è Diodoro di Sicilia, scrittore greco del I secolo. Egli ritiene che gli eroi greci avrebbero un'origine egizia, compreso Cadmo, Aegyptus, Danaus e altri.

Ora, un'analisi approfondita rivela che Diodoro mai svolse ricerche servendosi di una rigorosa metodologia storica: « semplicemente, riportò ciò che i sacerdoti egizi gli riferivano quando visitò quel paese durante le 180 Olimpiadi (60-56 a.C.) ». ${ }^{61}$ Inoltre, le deduzioni di Diop si fondamentano non tanto su fatti storici, ma in miti: il racconto che Diodoro fa di Danaus è un mito, che inizia con l'espulsione di Io (bis-bis nonna di Danaus) da parte di Zeus a causa della gelosia di sua moglie Era, e termina col ritorno di Danaus e le sue 50 sorelle a Argo, città greca.

Concludendo, non esistono prove decisive che dimostrino che la civiltà egizia era nera, anzi, nonostante grandi zone di ambiguità, le fonti sembrerebbero confermare probabilmente il contrario, ossia, che nel mondo antico esisteva una distinzione fra Egizi e Africani, avvicinando i primi maggiormente al Vicino Oriente che all'Africa sub-sahariana. Infine, il tentativo, da parte degli afrocentristi, di scambiare il mito per una narrativa storica è un metodo che non può essere accettato scientificamente.

\subsection{L'importanza degli Egizi nella costruzione dell'unità culturale africana}

Come si è spiegato sinteticamente sopra, il diffusionismo costituisce l'idea centrale per l'edificazione di un concetto unitario di Africa e, quindi, di cultura africana. Molti critici hanno rivolto la loro attenzione proprio a questa idea di un diffusionismo egittocentrico che si sarebbe espanso verso tutto il resto del continente africano.

Secondo Howe, la maggior parte dei gruppi etnici presenti oggi nell'Africa Australe sarebbe il prodotto finale di movimenti demografici assai recenti (secolo XVIII) e spesso violenti, conseguenza delle incursioni coloniali e dell'espansiione Zulu. ${ }^{62}$

\footnotetext{
${ }^{60}$ Cf. F.M. SNOwDEN, « Bernal's "Blacks” and the Afrocentrists », 1996.

${ }^{61}$ M. LeFKOWITZ, Not Out of Africa, 1996, p. 20.

${ }^{62}$ S. Howe, Afrocentrism, p. 147.
} 
Le migrazioni richiamate da Diop e Asante non avrebbero, perciò, sufficiente sostegno di tipo storico. Howe ritiene anche che il continente africano sia noto per non aver mai avuto un grande stato nazionale centrale, bensì entità politiche fortemente decentrate. ${ }^{63}$ La tesi del diffusionismo porterebbe inoltre gli afrocentristi a commettere altri errori di carattere storico, ad esempio avanzando l'idea della sequenzialità fra civiltà nubiana e egiziana, mentre è ormai noto che esse convissero lungamente nello stesso spazio temporale. Nessuna fonte archeologica corrobora questa idea degli afrocentristi.

Altre evidenze storiche oggi disponibili - oltre a quelle appena ricordate - sembrano rafforzare le posizioni dei critici dell'Afrocentrismo. In ambito commerciale, i flussi erano molto più frequenti da sud verso nord che viceversa, nonostante gli scambi fossero molto scarsi, in termini assoluti, almeno fino all'introduzione del cammello come mezzo di trasporto, in epoca cristiana. Secondo Howe, «... c'è assai poca - o addirittura nessuna - base, nella maggioranza del territorio africano, per difendere l'idea - fondamentale per la mitografia afrocentrica - che le società storicamente conosciute del continente affondino le sue origini in movimenti demografici massicci e di lunga distanza, al di fuori della Valle del Nilo ». ${ }^{64}$ La tesi del diffusionismo egittocentrico sembra quindi poggiare su basi storiche assai labili.

Parallelamente e conseguentemente al diffusionismo, l'idea della supremazia egizia in termini di civiltà continua nell' « unanimismo ». Secondo questo concetto, esisterebbe un'unità assoluta della cultura africana, derivante dall'Egitto. Le questioni principali qui affrontate hanno a che vedere con: 1. « egittocentrismo »; 2. unità linguistica e culturale africana; 3. Somiglianza fisica e genetica fra gli antichi Egizi e gli africani attuali.

1. «Egittocentrismo » e «Mito » dell'Origine dell'Antico Egitto

Il mito dell'Egitto come culla dell'umanità e dell'unità culturale africana fu sostenuto da vari autori, prima che l'Afrocentrismo ne facesse un suo vessillo. Importanti esponenti massonici usarono questo "misterioso » Egitto per farne una delle basi del loro pensiero e delle loro pratiche.

Nel 1859 - ricorda Howe - John Taylor aveva sostenuto l'idea di un Egitto " segreto », a partire dall'origine divina delle piramidi; ma è soprattutto da Piazzi Smith che questa idea si rafforza. Perfino due pilastri del pensiero afro-americano,

\footnotetext{
${ }^{63}$ IDEM, p. 146.

${ }^{64}$ IDEM, p. 147.
} 
William Trotter e Booker T. Washington, aderirono alla Prince Hall Grand Lodge di Boston, divenendo l'elemento di collegamento fra Egitto misterioso dei massoni e incipiente pensiero africano (e più tardi afrocentrico).

Fu così che, almeno negli Stati Uniti, il «mito » dell'Egitto misterioso si formò: agli afrocentristi fu sufficiente incanalarlo all'interno delle loro teorie, facendo così riferimento a una tradizione ben consolidata. Direttamente legato a questo « mito » è l'altro, relativo alla superiorità della razza nera, che trova in Egitto la sua manifestazione massima. Il mito del « Rastafari », per esempio, ritiene che i primi etiopi scesero dall'Egitto, e da qui verso Grecia e Roma, influenzando così la cultura europea e garantendo l'unità «nera » di quella africana. Marcus Garvey (primo eroe della Giamaica) è colui che sostenne queste idee, secondo una teoria deterministica delle razze, in cui l'Africa guidò il mondo, ma poi perdette il primato che adesso può recuperare.

2. Unità linguistica e culturale africana

La nozione di identità razziale in seno al « popolo africano », derivante dalla teoria del diffusionismo e così cara a Asante, presuppone l'idea di una cultura, una base linguistica e un'etica comuni. È soprattutto l'allievo diretto di Diop, Obenga, a postulare la somiglianza fra le varie lingue africane e fra queste e l'antico idioma egizio. Tale operazione viene da lui condotta cercando di fornire dimostrazioni pratiche (come la radice di diverse parole) e deducendo un'unità linguistica generale all'interno del continente africano. ${ }^{65}$ Ricerche di vari autori dimosterebbero che l'origine delle lingue bantu non dovrebbe essere ricercata nella Valle del Nilo, ma in Nigeria e in Cameroun. ${ }^{66}$

Nonostante sia molto probabile che l'origine del linguaggio, come del resto della specie Homo Sapiens, debba essere ricercata in Africa, sembra che una significativa differenziazione linguística sia occorsa nel tempo, fatto che avrebbe determinato la compresenza di differenti famiglie linguistiche nel continente più ricco da questo punto di vista (circa il $30 \%$ del patrimonio linguistico attuale si concentra qui), smentendo quindi l'idea di un'unità monolitica, così come postulato dagli afrocentristi.

I gruppi fino a oggi identificati sono i due principali, il NiloSahariano e il Nigero-Cordofano, più 1'Afro-asiatico e il Khoisan, parlato nella parte sud-occidentale del continente. ${ }^{67}$

\footnotetext{
${ }^{65}$ T. OBENGA, La philosophie africaine, 1990.

${ }^{66}$ Cf. J. VAnsina, Paths in the Rainforest, 1990.

${ }^{67}$ A. FERRARI, Lingue africane, 2011.
} 
Studi ancora più recenti, condotti da Quentin Atkinson e riportati dalla stampa internazionale, dimostrerebbero che l'origine del linguaggio sarebbe localizzata nell'Africa sudoccidentale, secondo ricerche basate non soltanto sulle parole, ma sui fonemi. Il « click» (ovvero, la presenza, in una lingua, di consonanti allusive non polmonari) è presente soprattutto nel continente africano (con più di 100 fonemi), e diminuisce proporzionalmente alla distanza dal « centro »: così, in Oceania esistono soltanto 13 click, nell'inglese $45 .^{68}$

Tali conclusioni mettono in dubbio non soltanto il primato dell'Egitto in questo importante ambito, ma anche l'origine stessa del linguaggio in Egitto, indebolendo ulteriormente la teoria dell'unità linguistica africana su basi egittocentriche.

3. Somiglianza fisica e genetica fra gli antichi Egizi e gli africani attuali

L'altro argomento rilevante usato dagli afrocentristi per dimostrare la supposta unità razziale e culturale del popolo africano si basa su una ipotetica comunanza di tipo genetico, incentrata sui tratti fisici esteriori maggiormente visibili (a partire dal colore della pelle). Come appena visto, tuttavia, tale patrimonio genetico comune sarebbe basato su rilevanze essenzialmente pittoriche e, in secondo luogo, linguistiche, assai incerte dal punto di vista dell'affidabilità storica.

Il movimento afrocentrico contemporaneo ignora, di fatto, le recenti - anche se non ancora decisive - scoperte dell'antropologia fisica, che presentano ulteriori elementi in grado di smentire i costrutti ideologici relativi all'unità africana, sia genetica che culturale. Ad esempio, analisi di tipo biochimico e fossile rivelerebbero che perfino fra Egizi e Nubi non vi sarebbe quella unità fisica che Diop e, successivamente, Asante, darebbero per scontata: i Nubi sarebbero assai diversi da 6 dei 12 gruppi di fossili egizi con cui sono stati comparati, così come il materiale fossile di Afalu e Tafaralt (Nord Africa) « had no similarity with Sub-Saharan Africa $»{ }^{69}$ I Somali, poi, sarebbero più simili agli Europei che alle popolazioni dell'Africa Occidentale e del Bacino del Congo, mentre somiglianze craniche sarebbero evidenti fra Egizi del periodo pre-dinastico e popolazioni indiane, dravidiche e vedda. ${ }^{70}$ Così concludono gli autori: "The argument over the 'racial' identity of the ancient Egyptians appears to have been fuelled more by

\footnotetext{
${ }^{68}$ N. WADE, Phonetic Clues Hint Language Is African-Born, 2011.

69 C.L. Brace, D.P. TraCER et AliI, «Clinn and Clusters Versus 'Race' », 1993, p. 15.

${ }^{70}$ IDEM, p. 23.
} 
'racial' pride than by any kind of objective assessment $»{ }^{71}$ Niente può essere detto in merito al colore della pelle degli Egizi, né che fossero neri, né che fossero bianchi. Qualsiasi deduzione in uno dei due sensi è perciò una conclusione puramente arbitraria.

Ancora più di recente, scoperte di antropologia fisica dimostrerebbero l'esistenza di almeno due diversi gruppi umani, dal punto di vista genetico, all'interno dello stesso territorio egizio pre-dinastico, uno nell'Alto Egitto, perfettamente sovrapponibile alle popolazioni del sud del continente, e l'altro nel Basso Egitto. La stessa formazione dello Stato egizio dovrebbe essere spiegata dall'azione di forze interne, associate a migrazioni su piccola scala, che avrebbero quindi contribuito ulteriormente a una diversificazione dei tipi umani in quel territorio. $^{72}$

Tali, recenti scoperte non hanno certamente messo la parola fine a una querelle ormai annosa, e che l'Afrocentrismo ha riacceso e reso attuale in ambito storico, filosofico, antropologico e linguistico. Tuttavia, esse hanno chiarito ulteriormente come gli afrocentristi, giocando sull'incertezza di risultati ancora provvisori, ma che comunque fanno propendere almeno per una evidente diversificazione genetica e dei tipi umani in Africa sin dal periodo pre-dinastico egizio, abbiano edificato un costrutto ideologico dalle fondamenta piuttosto labili anche se assai attraenti e stimolanti dal punto di vista ideologico.

\subsection{Le critiche allo "Stolen Legacy"}

L'idea dello "stolen legacy » è una conseguenza logica e radicale del diffusionismo e dell'egittocentrismo teorizzato dall'Afrocentrismo. L'argomento in favore dello «stolen legacy » viene svolto mediante due approcci: il primo (che non sarà qui approfondito) è di tipo «indiretto », ossia basato sulla comparazione fra pensiero filosofico e scientifico egizio e greco. Gli elementi essenziali sono stati dati nel punto precedente, e sarebbe qui troppo lungo discutere su possibili influenze del pensiero filosofico e scientifico egizio in rapporto a quello greco, vista anche la scivolosità della materia; il secondo (che costituirà l'analisi di questo punto), di tipo più «diretto », incentrato sull'analisi delle fonti storiche dell'epoca, le quali dimostrerebbero un rapporto stretto e costante fra le due civiltà, ivi compreso in ambito culturale.

\footnotetext{
${ }^{71}$ IDEM, p. 22.

72 S.R. ZAKRZEWSKI, «Population Continuity or Population Change. Formation of the Ancient Egyptian State », 2007.
} 
Fra le fonti allo scopo utilizzate dal movimento afrocentrista, a partire dall'opera di George James, vi sono anzitutto quelle greche. Una delle principali è rappresentata da Erodoto, i cui scritti sono considerati del tutto attendibili. Al contrario, i critici dell'Afrocentrismo ritengono che Erodoto non possa essere considerato alla stregua di uno storico credibile, poiché mescola costantemente narrazione letteraria e storiografica, è influenzato da una visione «religiosa» della storia, coerentemente con l'ambiente culturale greco del secolo V a.C., infine è solito fornire versioni pro-elleniche di alcuni episodi centrali, come quello relativo al regno di Cambise in Egitto (522-525 a.C.), che pongono seri dubbi su tutta la sua opera. ${ }^{73}$ Secondo Mary Lefkowitz, Erodoto «deliberatamente inventò alcune delle disinformazioni nella sua narrativa ${ }^{74} \mathrm{e}$, nonostante Diodoro cerchi di distinguere fra fiction e realtà storica, egli usa sempre fonti greche, mai egizie, di testimoni orali che, ovviamente, non possono essere considerati attendibili. Da una simile impostazione, Martin Bernal ritiene che il culto di Dioniso venga da quello di Osiris. Attraverso quali prove? Ancora una volta, la fonte è Erodoto, che giunge a questa conclusione a partire dal fatto che nei due culti c'è un pene preso da una donna durante il rituale. Visto che quella egizia è una civiltà più antica rispetto a quella greca, se ne deduce che fu la seconda a «bere » dalla prima. Si usa il principio "post hoc ergo propter hoc», senza alcun'altra prova effettiva per corroborare la tesi dello « stolen legacy ».

Infine, altre due inesattezze di cui James e Bernal sono accusati hanno a che vedere con: 1. l'influenza egizia nel pensiero aristotelico. Secondo questa ipotesi, lo Stagirita avrebbe tratto gran parte delle sue conoscenze dalla Biblioteca di Alessandria. Tuttavia, questa fu costruita e riempita di volumi soltanto dopo la morte del filosofo greco, quindi egli non usò certamente $\mathrm{i}$ testi egizi là raccolti; 2 . I «miti » antichi come fonti storiche: Bernal è continuamente accusato di fare un uso inappropriato, appunto storico, dei miti, ricevendo così dure critiche di carattere metodologico che pregiudicano il suo intero lavoro e, naturalmente, anche le sue conclusioni.

Attraverso questi esempi è possibile comprendere l'importanza del trattamento delle fonti per fondamentare una teoria di tipo ideologico e filosófico.

Sarà sulla base di simili (pseudo)certezze che Asante comincerà a costruire una tradizione afrocentrica, ispirandosi a Anta Diop, James e Bernal. Il dibattito che egli ha scatenato è stato caratterizzato da una elevata dose di ideologia, cosicché i

\footnotetext{
${ }^{73}$ M.T. JOÃO, « Heródoto e o Testamento de Udjahor-Resenet », 2005.

${ }^{74}$ M. LEFKOWITZ, Not out of Africa, p. 58.
} 
suoi critici hanno sempre cercato di riportare il discorso su un piano di maggiore attendibilità e verificabilità storica delle fonti, a partire da quelle egizie, che rappresentano il cuore della teoria afrocentrica. La rivalutazione del pensiero africano probabilmente non avrebbe necessitato di una costante forzatura delle fonti, operazione, questa che, al contrario, rischia di pregiudicare quanto di buono, in termini di contenuto, l'Afrocentrismo ha proposto sullo scenario filosofico contemporaneo.

\section{Riflessioni conclusive}

Il complesso dibattito appena descritto costituisce una piccola porzione della più generale e appassionata discussione intorno ai temi posti alla ribalta dall'Afrocentrismo. Ha probabilmente ragione Obenga, quando afferma, rispondendo direttamente a Mary Lefkowitz: « È la filosofia hegeliana della storia che sta in causa $»{ }^{75}$ Sulla scia di quanto Diop aveva sostenuto a suo tempo, il punto centrale non è tanto l'accertamento delle fonti, quanto la contestazione, a qualsiasi costo, dell'antica visione hegeliana, che negava la possibilità della storia agli Africani. E così continua: «Rompendo il paradigma hegeliano, i ricercatori africani stanno realizzando una grande rivoluzioneo intellettuale, storiografica e filosofica. Mary Lefkowitz fa di tutto per ritardare questa rivoluzione e la Rinascita Africana $»{ }^{76}$

In questo complesso gioco di posizionamento, lo storico svolge un ruolo fondamentale: l'analisi attenta delle fonti costituisce probabilmente l'unico ancoraggio sicuro per interpretare corretamente i principali elementi della civiltà egizia e dei rapporti che questa ha intrattenuto coi suoi vicini così come coi popoli europei, quali influenze essa abbia trasmesso agli uni e agli altri, quali elementi di continuità e quali innovazioni i Greci hanno introdotto rispetto ai popoli che li avevano preceduti. Ma, per fare questo, occorre non dimenticare mai una grande lezione che un noto storico africano aveva dato negli anni Sessanta: « La storia africana non necessita di regole speciali, di 'invenzioni', di idealizzazioni romantiche, di pie illusioni. Quello che è necessario è lo stesso che è necessario per qualunque altro tipo di storia, la verità, in maniera tanto completa quanto essa può essere ragionevolmente determinata $»{ }^{77}$

\footnotetext{
${ }^{75}$ T. OBENGA, Le sens de la lutte contre l'afrocentrisme eurocentrique, 2001, p. 50.

${ }^{76}$ IDEM, p. 51.

${ }^{77}$ C. Williams, Problems in African History, 1964, p. 6.
} 
È proprio questa idealizzazione - che incide direttamente nella lettura delle fonti egizie - che fa in modo che gli afrocentristi leggano il modello sociale e filosofico egizio senza evidenziare le zone di ombra che esso implica: a partire dall'esaltazione di una società (quella egizia) profondamente diseguale e basata in larga misura sulla schiavitù, e supportata da un'ideologia che giustifica un simile ordine sociale.

Se il dibattito succintamente descritto ha messo in evidenza soprattutto questioni inerenti alle fonti storiche e al suo uso, discorso a parte andrebbe fatto per quel che riguarda la filosofia egizia e i suoi rapporti con quella greca. In questo senso, la discussione è ancora a un livello assai più acerbo rispetto a quello di tipo storico, e tuttavia almeno due osservazioni generali possono essere compiute: in primo luogo, l'Afrocentrismo ha ridestato l'attenzione della comunità internazionale sulle origini del pensiero filosofico e scientifico. E ciò rappresenta un indubbio merito di questa corrente, scuotendo quelle che ormai da più di due millenni erano considerate certezze assolute e inattaccabili, quasi dogmatiche. In secondo luogo, l'eccesso ideologico con cui l'Afrocentrismo ha riproposto una simile tematica ha reso poco credibile un tentativo intellettuale comunque coraggioso e interessante. Se, infatti, esistono parti del pensiero filosofico e scientifico che probabilmente gli Egizi avevano elaborato prima dei Greci, in altri casi ciò è semplicemente indimostrabile e, probabilmente, impossibile. Tutta la filosofia politica, che trova sviluppo all'interno del modello democratico ateniese, non avrebbe avuto alcuna possibilità di emergere in Egitto, dove l'ordine sociale era non soltanto gerarchizzato, ma addirittura (come evidenziato dallo stesso Diop) basato su caste. Infine, anche ammettendo la capacità, da parte degli Egizi, di elaborare una vera filosofia (ad esempio della natura o di tipo etico), le argomentazioni con cui l'Afrocentrismo costruisce la teoria dello Stolen Legacy sono deboli e francamente non conclusive.

Tutti questi aspetti meriterebbero pertanto un maggiore approfondimento, concentrando l'analisi, questa volta, sui contenuti effettivi del pensiero filosofico e scientifico delle due maggiori civiltà dell'antichità pre-cristiana. 
Abstract

Questo articolo intende trattare di una delle proposte maggiormente controverse da parte della corrente afrocentrica: le origini africane (egizie) del pensiero filosofico, in aperta opposizione con la teoria classica, definita da tale corrente come eurocentrica o del "miracolo greco». Una simile interpretazione serve ai suoi fondatori, fra cui Diop, Obenga e Asante, per cercare di dimostrare l'infondatezza delle tesi opposte, originate da puri pregiudizi razziali, stratificati da più di due millenni di dominio della cultura occidentale neiconfronti delle altre. Tali pregiudizi, culminati con le note posizioni di Hegel ma poi sviluppatisi ulteriormente in epoca più moderna, avrebbero infatti avuto la forza di negare le origini egizie del pensiero filosofico astratto, così come di quello scientifico, giungendo a formulare una vera e propria teoria dello Stolen Legacy (George James). L'articolo, più che entrare nel merito di tale dibattito, ha come obiettivo principale quello di mostrare come le fonti storiche dell'Antico Egitto siano state interpretate in modo sostanzialmente opposto da parte degli afrocentrici in relazione alle letture tradizionali, suscitando infinite polemiche, senza giungere, sino ad oggi, ad alcun consenso. Il dibattito che ne è scaturito ha dato luogo a evidenti forzature delle fonti egizie, usate per comprovare posizioni ideologiche e filosofiche che hanno molto a che fare con l'egemonia culturale a livello mondiale, ma pochissimo col corretto uso della storia.

Parole-chiave: Afrocentrismo, Eurocentrismo, Fonti storiche, Egitto, Filosofia 
Abstract

This article attempts to shed light on one of the most controversial proposals formuled by the Afrocentric philosophical movement: the African (Egyptian) origins of the philosophical thought, in open opposition to the classic theory, defined as Eurocentric or of the «Greek miracle». Such interpretation serves to its founders, among which Diop, Obenga and Asante, to try to show the groundlessness of the opposite theses, originated by pure racial prejudices, stratified by more than two millennia of domination of Western culture towards all the others. These prejudices, which culminated in the known positions of Hegel but developed further in a more modern era, would have been able to deny the Egyptian origins of the philosophical abtract thought, as well as of the scientific one, arriving to formulate an actual theory of the Stolen Legacy (George James). The article, more than enter this debate, has, as its main objective, to show how the historical sources of Ancient Egypt have been interpreted in an opposite way by the Afrocentrics in relation to the traditional readings, arousing endless controversy but without reaching, until today, any consensus. The dabate that followed provoked evident forcing of the Egyptian sources, used for proving ideological and philosophical positions strictly related to cultural hegemony on a global scale, but very few to the correct use of history.

Keywords: Afrocentricity, Eurocentrism, Historical sources, Egypt, Philosophy 


\section{BIBLIOGRAFIA}

Adams W., Nubia: Corridor to Africa, Princeton, New York, 1977.

ApPIAH K.A., In My Father's House: Africa in the Philosophy of Culture, Methuen, London, 1992.

ASAnTe M.K., Afrocentricity. The Theory of Social Change, African American Images, Chicago, $1980\left(2003^{2}\right)$.

Asante M.K., The Afrocentric Idea, African American Images, Chicago, 1987.

Asante M.K., Kemet, Afrocentricity and Knowledge, Africa World, Trenton, 1990.

Asante M.K., The Egyptian Philosophers, African American Images, Chicago, 2000.

ASANTE M.K., «An African Origin of Philosophy: Myth or Reality? », in City Press, July 2004, consultato 1'8/11/ 2015 in www.asante.net/articles/26/afrocentricity.

BAngurA A.K., «From Diop to Asante: Conceptualizing and Contextualizing the Afrocentric Paradigm » in The Journal of Pan African Studies 5, 1, March 2012, pp. 103-125.

Bernal M., Black Athena, Rutger University Press, London, 1987.

Brace C.L., Tracer D.P. et AliI, « Clinn and Clusters Versus 'Race': A Test in Ancient Egypt and the Case of a Death on the Nile » in Yearbook of Physical Anthropology 36 (1993), pp. 1-31.

BussotTi L., Afrocentrismo e questione religiosa, in GATTI, Bussotti, Nhaueleque, Africa Afrocentrismo e Religione, Aviani, Udine, 2010.

Coon C.S., The Race of Europe, Mac Millan, London, 1939

Coon C.S., The Origin of Races, Knopf, New York, 1962

Crook P., Grafton Elliot Smith, Egyptology \& the Diffusion of Culture, Sussex Academic Press, Brighton-Portland-Toronto, 2012.

Diop C.A., The African Origin of Civilization, Lawrence Hill Books, Chicago, 1974.

DioP C.A., Precolonial Black Africa, Lawrence Hill \& Company, Westport, 1987.

EwING S.D. (2006), Sesostris the Great, the Egyptian Hercules, in http://www.africaresource.com/rasta/sesostris-the-greatthe-egyptian-hercules/sesostris-the-great-the-egyptianhercules/, consultato il 26/08/2015.

FANON F., Peau noire, masques blancs, Seuil, Paris, 1952. 
FERRARI A., Lingue africane, MISNA, Dossier n. 6, Marzo 2011, in www.misna.org, consultato il 27/08/2015.

Hobsbawm E. \& RANGer T. (Eds.), The Invention of Tradition, Cambridge University Press, Cambridge, 1983.

Howe S., Afrocentrism. Mythical Pasts and Imagined Homes, Verso, London, 1988.

JAMES G., Stolen Legacy, Library of Alexandria, 1954 (1989²).

JOAO M.T., «Heródoto e o Testamento de Udjahor-Resenet », in Revista Cantareira - Revista Eletrônica de História 2, 3, 3, dic. 2005, in: hhttp://www.historia.uff.br/Cantareira.

Karenga M., MAAT. The Moral Idea of Ancient Egypt, Routledge, New York, 2004.

Keyta M., Race and the Writing of History: Riddling the Sphinx, Oxford University Press, New York, 2000.

LefKowitz M., Not Out of Africa. How Afrocentrism became an excuse to teach myth and history, Basic Books, New York, 1996.

Moret A. \& Davy G., From Tribe to Empire, New York, Cooper Square Publisher, 1970.

Morton S.G., Crania Aegyptiaca; or, Observations on Egyptian ethnography, derived from anatomy, history, and the monuments, J. Penington, Philadelphia, 1844.

NJAMI-Nwadi S.B., «Origine Africaine des principales religions révélées ", in Les Dieux et l'Afrique, in Nomade 4 (1993), pp. 8-17.

OBENGA T., «The genetic linguistic relationship between Egyptian (ancient Egyptian and Coptic) and modern NegroAfrican language " in UNESCO, The peopling of ancient Egypt and the deciphering of Meroitic script, Proceedings of the symposium held in Cairo from 28 January to 3 February 1974, Paris, UNESCO, 1978, pp. 65-71.

OBENGA T., La philosophie africaine de la période pharaonique. 2780-330 avant notre ère, L'Harmattan, Paris, 1990.

OBEngA T., Le sens de la lutte contre l'afrocentrisme eurocentrique, Khepera-L'Harmattan, Paris, 2001.

PATterson T.C. (Org.), Making Alternative Histories: the practice of Archaeology and History in non-Western setting, School of American Research Press, Santa Fe, 1982.

Quack J.F., Chauveau M. (2001), Scienza Egizia. La conoscenza normativa, em www.treccani.it, consultato il 21/03/2014. 
RigGS C., Unwrapping Ancient Egypt, Bloomsbury Academic, London-New York, 2014.

SENTWALI BAKARI R., "Epistemology from an Afrocentric Perspective: Enhancing Black Students' Counsciousness through an Afrocentric Way of Knowing " in Different perspectives on Majority Rules, 20 (1997), consultato il 30/04/2014 http://digitalcommons.unl.edu/pocpwi2/20.

SINORITA FIERRO R., L'identità afroamericana, L'Harmattan Italia, Torino, 2001.

SNOWDEN F.M., "Bernal's "Blacks" and the Afrocentrists » in LefKowitz M., MAC LEAN Rogers G. (Eds.), Black Athena Revisited, The University of North Carolina Press, USA, 1996.

UNESCO, Foreword: Preparation of a General History of Africa, in UNESCO, The peopling of ancient Egypt and the deciphering of Meroitic script, Proceedings of the symposium held in Cairo from 28 January to 3 February 1974, Paris, UNESCO, 1978.

UNESCO, Symposium on the Peopling of Ancient Egypt, $A$ Report on the discussion, in UNESCO, The peopling of ancient Egypt and the deciphering of Meroitic script, Proceedings of the symposium held in Cairo from 28 January to 3 February 1974, Paris, UNESCO, 1978.

VAnsina J., Paths in the Rainforest: Toward a History of Political Tradition in Equatorial Africa, University of Wisconsin Press, Madison, 1990.

Van Wick Smith M., The First Ethiopians, Wits University Press, Johannesburg, 2009.

WADE N., "Phonetic Clues Hint Language Is African-Bor », in The New York Times, 14/04/2011, consultato il 27/08/2015 http://www.nytimes.com/2011/04/15/science/15language.html

Williams C., Problems in African History: A College Lecture Series, Washington, 1964.

ŽÁBA Z., Les Maximes de Ptahhotep, Éditions de l'Académie Tchécoslovacque des Sciences, Prague, 1956.

ZAKRZEWSKI S.R., «Population Continuity or Population Change. Formation of the Ancient Egyptian State ", American Journal of Physical Anthropology 132 (2007), pp. 501-509. 